\title{
LIFR increases the release of soluble endoglin via the upregulation of MMP14 expression in preeclampsia
}

\author{
Hua $\mathrm{Li}^{1}$, Julei $\mathrm{Yao}^{1}$, Xinwen Chang ${ }^{1}$, Jinting $\mathrm{Wu}^{1}$, Tao Duan ${ }^{1,2}$ and Kai Wang ${ }^{1}$ \\ ${ }^{1}$ Clinical and Translational Research Center, Shanghai First Maternity and Infant Hospital, Tongji University School of \\ Medicine, Shanghai, People's Republic of China and ${ }^{2}$ Department of Obstetrics, Shanghai First Maternity and Infant \\ Hospital, Tongji University School of Medicine, Shanghai, People's Republic of China
}

Correspondence should be addressed to T Duan or KWang; Email: tduan@yahoo.com or kaiwangcn@yahoo.com

\begin{abstract}
Preeclampsia (PE) is a pregnancy-specific disorder that is the main cause of maternal and perinatal morbidity and mortality worldwide. Inadequate trophoblastic invasion and endothelial dysfunction in the placenta are considered the foundation of the pathogenesis of preeclampsia in which soluble endoglin (sENG) plays an antiangiogenic role in the development of PE. The leukemia inhibitory factor receptor (LIFR) has been widely studied and is highly involved in arterial injury in vivo and in the migration of cancer cells in vitro. Here, we tested the hypothesis that LIFR may be correlated with preeclampsia through its regulation of the release of sENG. Our data showed that LIFR protein, the expression of which significantly decreased with the progression of pregnancy, was located in the syncytiotrophoblast and cytotrophoblast. The LIFR protein level was increased in pregnancies with preeclampsia compared with normotensive full-term pregnancies. After the overexpression of LIFR in HTR8/SVneo cells, the release of sENG as well as the migration and invasion were significantly enhanced. Moreover, we also observed that LIFR induced the expression of matrix metalloproteinase14 (MMP14) and that the knockdown or inhibition of MMP14 decreased the release of sENG, as well as increased the LIFR-induced migration and invasion of HTR8/SVneo cells. These studies demonstrated that LIFR promoted the release of sENG through MMP14 in vitro, which indicates that LIFR may be involved in the development of preeclampsia. Reproduction (2018) 155 297-306
\end{abstract}

\section{Introduction}

Preeclampsia, which is characterized by hypertension and proteinuria after 20 weeks of gestation, affects $5-8 \%$ pregnancies and results in substantial maternal and neonatal complications and death, especially in low-income and middle-income countries (Redman \& Sargent 2005, Hansen 2010, Souza et al. 2013, Mol et al. 2016, Ho 2017). However, its pathogenesis and pathophysiology are poorly understood. It is well known that excessive levels of placenta-derived antiangiogenic factors in the circulation such as soluble endoglin (sENG) (Levine et al. 2006, Venkatesha 2006), and soluble fms-like tyrosine kinase 1 (sFLT1) (Zeisler et al. 2016, McGinnis et al. 2017) may cause endothelial dysfunction in preeclampsia. The concentration of sENG in the maternal circulation in cases of preeclampsia was significantly increased and was positively correlated with the severity of preeclampsia (Venkatesha 2006, Jeyabalan et al. 2008). Furthermore, the release of sENG has been shown to be regulated by matrix metalloproteinase-14 (MMP-14, also known as MT1-MMP) in cases of vascular injury and tumor (Hawinkels et al. 2010, Gallardo-Vara et al. 2016), but little is known about the regulation of sENG release in PE.
The leukemia inhibitory factor receptor (LIFR), whose ligand LIF belongs to the IL- 6 family of cytokines, is important in the pregnancy process. The deletion of the LIFR gene in animals is so severe that LIFR-/- animals do not reach reproductive age; moreover, LIFR-/- fetuses experience placental, skeletal, neural and metabolic defects and die during the perinatal period (Ware et al. 1995). A previous study has demonstrated that LIF is correlated with the pathogenesis of preeclampsia as it promotes trophoblast invasion via urokinase-type plasminogen activator receptor (UPAR) (Zheng et al. 2016), which suggests that the LIF/LIFR system may play an important role in PE. However, the role of LIFR in preeclampsia remains to be revealed.

In this study, we hypothesize that LIFR is potentially involved in the development of preeclampsia as a result of its interference with the release of sENG. Therefore, we aim to determine LIFR expression during normal pregnancy and in patients with PE, as well as its effects on the release of $s E N G$, and on the migration and invasion of trophoblast cells (HTR8/SVneo). In addition, we also investigate the relationship between MMP14 and LIFR to further explore the possible mechanisms that are responsible for the altered LIFR expression in preeclampsia. 


\section{Materials and methods}

\section{Patients and sample collection}

Maternal blood samples from patients in different trimesters of pregnancy and from patients diagnosed with PE were collected in vacutainer tubes with EDTA for plasma separation. The samples were centrifuged at $4^{\circ} \mathrm{C}$ with a relative centrifugal force of $1500 \mathrm{~g}$ for $15 \mathrm{~min}$. The supernatant (plasma) samples were transferred to clean 1.5$\mathrm{mL}$ Eppendorf tubes and stored at $-80^{\circ} \mathrm{C}$ for ELISA (enzymelinked immunosorbent assay) analysis. Preeclampsia was defined according to the guidelines of the US National Institutes of Health (US National High Blood Pressure Education Program 2000) (Goldenberg et al. 2008). Placental tissues were obtained from both normal term control and PE patients. Samples were collected immediately $(<30 \mathrm{~min})$ after cesarean section, cut near the center zone of the fetal part of the placenta after the decidua and amniotic membranes were removed and were then washed with sterile PBS to remove maternal blood. The chorionic villi samples of the first trimester $(n=7)$ were collected immediately after vacuum aspiration and were rinsed with sterile PBS. All samples were frozen and stored in liquid nitrogen for Western blot. Additional placental tissues were embedded in paraffin for immunohistochemistry (IHC).

This study was approved by the Scientific and Ethical Committee of Shanghai First Maternity and Infant Hospital of Tongji University. Human samples were used according to the guidelines of the Scientific and Ethical Committee of Shanghai First Maternity and Infant Hospital of Tongji University. These samples were obtained with a written informed consent provided by the participants.

\section{Cell culture}

The human trophoblast cell line HTR-8/SVneo and the human choriocarcinoma cell line JAR were initially obtained from Dr Charles H. Graham (Queen's University, Ontario, Canada) as previously described (Wang et al. 2008, Dai et al. 2011). HTR-8/SVneo and JAR cells were cultured in DMEM/F12 supplemented with $10 \%$ fetal bovine serum and $1 \%$ penicillin/ streptomycin and were incubated at $37^{\circ} \mathrm{C}$ in a humidified atmosphere containing $5 \% \mathrm{CO}_{2}$. For proliferation assays, cells were incubated with different concentrations of recombinant LIF $(0,10,25,50 \mathrm{ng} / \mathrm{mL}$; R\&D Systems: 7734-LF) for $48 \mathrm{~h}$. For migration and invasion assays, cells were cultured with different concentrations of recombinant human LIF (same concentrations as those used in the proliferation assay), a STAT3 inhibitor ( $5 \mu \mathrm{m}$; Selleck: S7501) or an MMP14 inhibitor (50 $\mu$ m; NSC405020, Selleck: S8072) for $24 \mathrm{~h}$. For the Western blot analysis, cells were incubated with rhLIF $(10 \mathrm{ng} / \mathrm{mL})$, a STAT3 inhibitor $(5 \mu \mathrm{m})$ or an MMP14 inhibitor $(50 \mu \mathrm{m})$ for 15 min.

\section{Western blot analysis}

Total placental tissue lysates were prepared by homogenization as previously described (Chung 2004, Jiang et al. 2010). Cells grown in a monolayer were washed with PBS and harvested for protein in RIPA buffer supplemented with a protease and phosphatase inhibitor (Roche). The protein concentration was determined by BCA Protein Assay Kit (Thermo Scientific). Placental lysates $(30 \mu \mathrm{g})$ and cell lysates $(20 \mu \mathrm{g})$ were separated on $10 \%$ SDS-PAGE gels with wet transfer to polyvinylidene difluoride membranes (Millipore). After blocking (7\% milk in TBST, $0.1 \%$ Tween-20) at room temperature for $2 \mathrm{~h}$ on a vertical shaker, PVDF membranes were probed with antibodies against LIF (Santa Cruz Biotechnology: sc-1336, 1:200), LIFR (Santa Cruz Biotechnology: sc-659, 1:200), pSTAT3 Tyr705 (Cell Signaling: 9131,1:1,000), STAT3 (Cell Signaling: 9139, 1:1000), MMP14 (Abcam: ab51074, 1:2500) and $\beta$-actin (Proteintech: 1:2000, USA). Membranes were visualized using enhanced chemiluminescence reagents (Millipore), and all Western blots were quantified for adjusted relative density using Image $(\mathrm{NIH})$. Data were normalized to $\beta$-actin.

\section{Immunohistochemistry (IHC)}

To determine tissue localization of LIFR and LIF, immunohistochemistry was performed as previously described (Jiang et al. 2010). Paraffin-embedded sections of placental tissues were deparaffinized and rehydrated. The endogenous peroxidase activity of the tissues was quenched by immersing the tissue sections in $3 \% \mathrm{H}_{2} \mathrm{O}_{2}$ in methanol for $10 \mathrm{~min}$; nonspecific epitopes were then blocked by incubating the slides in $1 \%$ horse serum albumin for $20 \mathrm{~min}$. The tissue sections were probed with a goat anti-LIF antibody (Santa Cruz: sc-1336, 1:50) and a rabbit anti-LIFR antibody (Santa Cruz: sc-659, 1:50).

\section{Quantitative real-time PCR}

Total RNA was isolated from cells using TRIzol (Invitrogen). The concentration of RNA was measured by a spectrophotometer (ND 2000, Nanodrop Inc, Wilmington, DE, USA). A total of $1 \mu \mathrm{g}$ of RNA was reverse-transcribed using the SuperScript First-Strand cDNA System (Takara). The resulting cDNA was used as a template for quantitative real-time PCR (qRT-PCR), which was performed using SYBR Green Premix Ex Taq (Tiangen, Beijing, China) in an ABI Prism 7000 Sequence Detection System. The D-glyceraldehyde-3-phosphate dehydrogenase (GAPDH) was used as an endogenous control for gene expression analysis. The primer sequences for qRT-PCR analysis were shown in Table 1.

Table 1 The primer set in this study.

\begin{tabular}{ll}
\hline & Sequence $\left(5^{\prime}-3^{\prime}\right)$ \\
\hline hLIFR & \\
Forward & GTGACCCACAACACAACTCTG \\
Reverse & CACATTCCAAGGGCATATCTGAG \\
hMMP14 & \\
Forward & GGCTACAGCAATATGGCTACC \\
Reverse & GATGGCCGCTGAGAGTGAC \\
GAPDH & \\
Forward & TGGGCTACACTGAGCACCAG \\
Reverse & AAGTGGTCGTTGAGGGCAAT \\
\hline
\end{tabular}




\section{Cell proliferation}

For the cell proliferation assay, cells seeded in 96-well plates (3000 cells/well) were cultured in DMEM/F12 media for $48 \mathrm{~h}$. The number of cells was measured by MTT assay (Promega, G3580). Wells containing known cell numbers (0, 1000, 2000, 5000, 10,000, 20,000 or 40,000 cells/well; 6 wells/ cell density) were treated in a similar manner to establish standard curves.

\section{Cell migration and invasion}

Cell migration and invasion were evaluated using a 24-Multiwell BD Falcon FluoroBlok Insert System (8.0- $\mu$ m pores; BD Biosciences, San Jose, CA, USA). For the cell invasion assay, the upper chambers of the inserts were pre-coated with $100 \mu \mathrm{L}$ of a 1:20 dilution of Matrigel in DMEM/F12 medium with $10 \%$ FBS for $60 \mathrm{~min}$ at $37^{\circ} \mathrm{C}$. JAR $\left(5 \times 10^{4}\right.$ cells/well $)$ and HTR8/SVneo cells $\left(8 \times 10^{4}\right.$ cells/well $)$ in DMEM/F12 medium with $10 \%$ FBS were seeded onto the upper side of the Transwell inserts. The bottom wells of the chamber were filled with DMEM/F12 medium with $10 \%$ FBS prior to cell seeding. After incubation at $37^{\circ} \mathrm{C}$ with $5 \% \mathrm{CO}_{2}$ and $95 \%$ air for $24 \mathrm{~h}$, cells that had migrated to and invaded the bottom of the inserts were stained with calcein AM $(0.2 \mu \mathrm{g} / \mathrm{mL}$; Invitrogen, No. C3100MP) for $30 \mathrm{~min}$, examined and counted by fluorescence analysis (Nikon).

\section{Lentivirus production and stable cell lines}

To establish cell lines that stably overexpress LIFR, lentiviral packaging was performed by co-transfection of HEK 293T cells with pWPXL and pWPXL-LIFR (Addgene) using Lipofectamine 2000 (Invitrogen) as previously described (Luo et al. 2014). Viruses were harvested $48 \mathrm{~h}$ after transfection, after which viral titers were determined. HTR8/SV-neo cells were infected with $1 \times 10^{6}$ recombinant lentivirus-transducing units in the presence of $6 \mu \mathrm{g} / \mathrm{mL}$ polybrene.

\section{SiRNA knockdown of LIFR and MMP14}

HTR8/SVneo cells were transfected with LIFR siRNA or scrambled siRNA with SuperFect Reagent (Qiagen). HTR8/ SVneo cells overexpressing LIFR were transfected with MMP14 siRNA or scrambled siRNA in the same way. Treatments were applied for $48 \mathrm{~h}$ after which the cell media were collected for sENG ELISA and cells were harvested for mRNA and protein extraction.

\section{ELISA analysis}

Concentrations of human LIF in placental tissue lysates were determined by Quantikine Human LIF (R\&D Systems: DLF00). Concentrations of human sENG and human sFlt-1 in plasma samples of maternal blood and cell supernatants transfected with overexpression vectors were determined by Quantikine Human Endoglin/CD105 (R\&D Systems: DNDG00) and Quantikine Human sFlt-1 (R\&D Systems: DVR100B) assays, respectively. All immunoassays were measured in a Varioskan Flash spectral scanning multimode reader at $450 \mathrm{~nm} / 570 \mathrm{~nm}$ (Thermo Scientific).

\section{Statistics analysis}

Data are expressed as the mean \pm the standard error of the mean (S.E.M.) or the mean \pm standard deviation (S.D.) and were analyzed using SPSS 23.0 statistical analysis software (SPSS). The differences between groups were analyzed by Student's two tailed $t$-test when two groups were analyzed or by ANOVA if more than two groups were analyzed. A $P$ value $\leq 0.05$ was considered statistically significant.

\section{Results}

\section{Characteristics of patients with preeclampsia and normal controls}

The patient characteristics were outlined in Table 2 . No statistically significant differences were observed in the maternal age and gestational age of patients with PE and normal full-term pregnancies $(P>0.05)$. The diastolic blood pressure (DBP) and systolic blood pressure (SBP) of patients with PE were substantially higher than those of patients with normal pregnancies $(P<0.001)$. In contrast, patients with PE had lower fetal weights $(P<0.01)$ compared with normal controls. The proteinuria in patients with PE was $2(+)$, while normal pregnant women exhibited no proteinuria.

\section{LIFR was upregulated in the placentas of patients with PE}

We first investigated placental LIFR protein expression and/or distribution in the first trimester, in full-term pregnancies and in patients with PE by Western blot and found that the LIFR protein level was significantly

Table 2 The basic information of pregnant women.

\begin{tabular}{|c|c|c|c|}
\hline & First trimester $(n=7)$ & Full term $(n=11)$ & $\mathbf{P E}(n=11)$ \\
\hline Maternal age (years) & $27.3 \pm 2.8$ & $29.8 \pm 0.9$ & $30 \pm 1.4$ \\
\hline Gestation age (weeks) & $7.0 \pm 0.3$ & $38.8 \pm 0.3$ & $37.7 \pm 0.5$ \\
\hline Fetal weight (g) & & $3422.3 \pm 59.8$ & $2886.4 \pm 171.6^{* *}$ \\
\hline Systolic pressure (mmHg) & & $112.5 \pm 2.4$ & $146.5 \pm 3.1 * * *$ \\
\hline Diastolic pressure $(\mathrm{mmHg})$ & & $73.5 \pm 2.7$ & $97.6 \pm 1.4^{* * *}$ \\
\hline Proteinuria & & $(-)$ & $(++)$ \\
\hline Mode of delivery & & $\mathrm{CS}$ & $\mathrm{CS}$ \\
\hline
\end{tabular}

$* P<0.05,{ }^{*} * P<0.01,{ }^{* * *} P<0.001$ (value as mean \pm S.E.M.). 
decreased from the first to the third trimester of pregnancy; importantly, the level was higher in pregnant women with $\mathrm{PE}$ than in women with normal pregnancies (Fig. 1A, B, D and $F$ ). However, no significant differences were observed in the expression of LIF protein according to both Western blot and ELISA (Fig. 1A, B, C, E and G). These data indicated that LIFR expression played an important role in pregnancy progression and that it might be correlated with the pathological mechanism of preeclampsia.

IHC was performed to determine the localization of LIFR and LIF within the human placentas (Fig. 1H). Positive brownish staining for LIFR was observed in the endothelium, syncytiotrophoblast and cytotrophoblast in the first trimester. Meanwhile the syncytiotrophoblast and cytotrophoblast were immunolabeled for LIF in the first trimester. In placentas derived from both normal full-term controls and women with PE, LIFR and LIF were located in the syncytiotrophoblast.

\section{LIFR enhanced the release of $S E N G$, as well as the migration and invasion of HTR8/SVneo cells}

Since LIFR was more highly expressed in pregnancies with PE, we investigated whether upregulated LIFR was
A

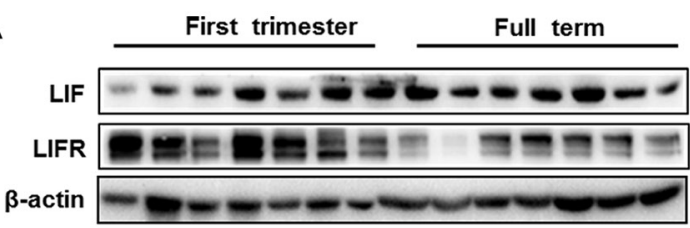

B

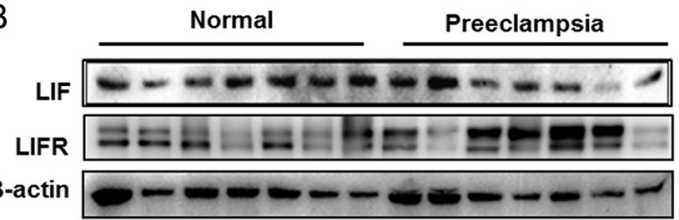

$E$

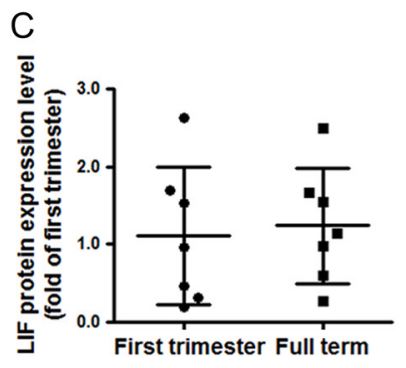

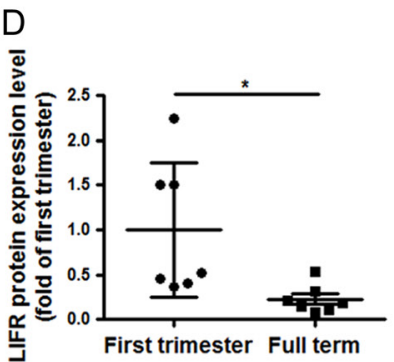

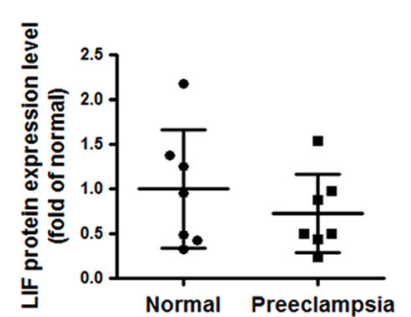

LIF

G

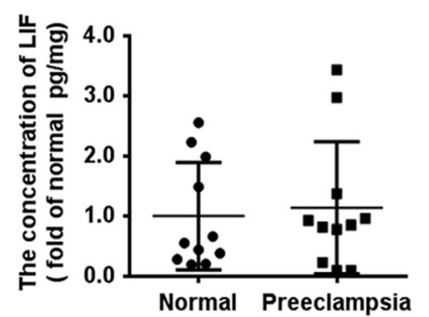

$\mathrm{H}$
First trimester

First trimester

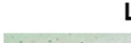

LIFR

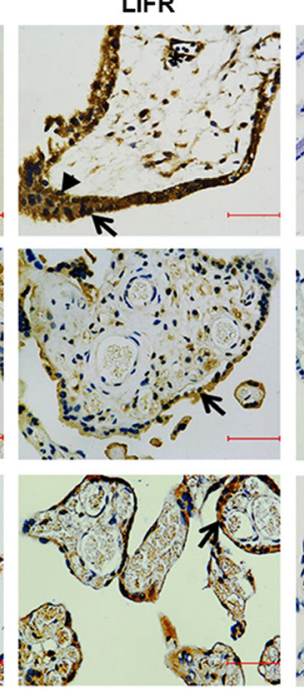

$\mathrm{F}$

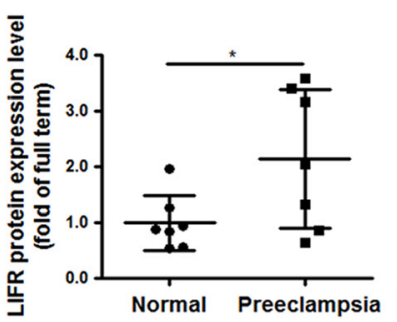

$\lg G$

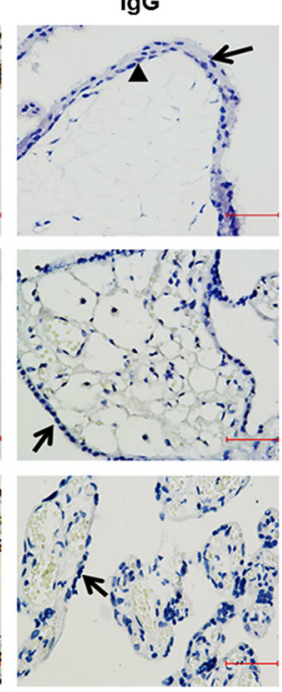

Figure 1 Expression levels of LIF and LIFR in human placentas from first trimester $(n=7)$, full term $(n=7 / 11)$ and preeclampsia $(n=7 / 11)$ was determined by Western blot, ELISA or immunohistochemistry. (A, C and D) The expression of LIF and LIFR protein in human placentas from the first trimester $(n=7)$ and full-term pregnancies $(n=7)$ was determined by Western blot. (B, E and F) The expression of LIF and LIFR protein in human placentas from pregnant women with preeclampsia $(n=7)$ compared with those from normotensive full-term pregnancies $(n=7)$ was determined by Western blot. (G) LIF protein in human placentas from women with preeclampsia $(n=11)$ and matched pregnant controls $(n=11)$ was measured by ELISA. (H) Immunohistochemical localizations of LIF and LIFR in normal and preeclamptic placentas. Arrows, syncytiotrophoblast; Arrowhead, cytotrophoblast; as-terisk, endothelium. Scale bar $=250 \mu \mathrm{m}$. Representative Western blots are shown for LIF, LIFR and $\beta$-actin. Data are normalized to $\beta$-actin and are expressed as the mean \pm S.D. (Student's $t$-test, $* P<0.05$ ). 


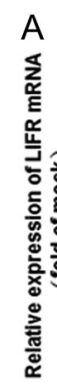

B
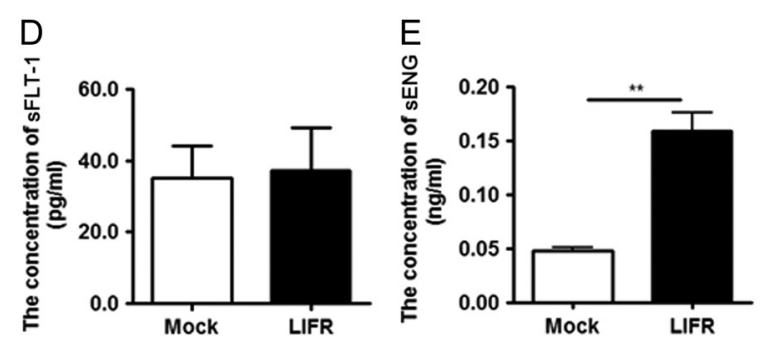
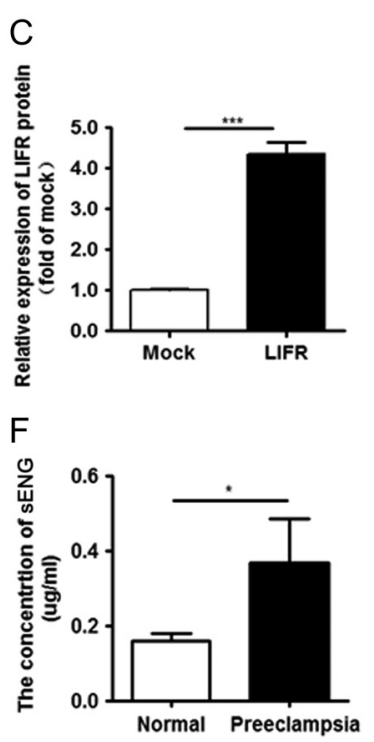

Figure 2 Overexpression of LIFR promotes the release of sENG in HTR8/SVneo cells. (A, B and C) The efficiency of the overexpression of LIFR was determined by qRT-PCR and Western blot. (D, E and F) The concentration of sENG and sFlt-1 in the cell supernatant after the overexpression of LIFR in HTR8/SVneo cells and in the plasma of women with PE or normotensive term pregnancies were measured by ELISA. Bars represent the means \pm S.E.M. (Student's $t$-test, ${ }^{*} P<0.05$, $\left.{ }^{* *} P<0.01,{ }^{* * *} P<0.001\right)$. involved in the release of sENG and sFlt-1 in PE. The efficiency of overexpression of LIFR was determined by qRT-PCR and Western blot (Fig. 2A, B and C). Our data showed that the concentration of sENG but not that of sFlt-1 in the cell supernatant was increased according to ELISA after the overexpression of LIFR in HTR8/SVneo cells (Fig. 2D and E). Moreover, we also observed that the concentration of sENG was significantly higher in the maternal plasma of patients with PE compared with normal controls (Fig. 2F). In addition, the overexpression of LIFR significantly enhanced cell migration and invasion (Fig. $3 \mathrm{~A}, \mathrm{~B}$ and $\mathrm{C}$ ) but did not alter the proliferation of HTR8/SVneo cells (Fig. 3D).

\section{LIFR-induced the expression of MMP14 in HTR8/SVneo cells}

It has been well demonstrated that MMP14 is correlated with the release of sENG and trophoblast invasion. To explore the involvement of MMP14 in the LIFR pathway, we first examined whether MMP14 expression could be regulated by LIFR. As shown in Fig. 4A, B and C, LIFR could increase MMP14 expression at both the mRNA and protein levels in HTR-8/SVneo cells. We also found that MMP14 expression was increased in the placentas of women with preeclampsia (Fig. 4D and E). In addition, we investigated the effects of knockdown LIFR on MMP14 expression and sENG release in HTR8/SVneo cells. After knockdown of LIFR by siRNA, the mRNA and protein expressions of MMP14, as well as sENG release were decreased, but not reach the statistical significance (data not shown). We speculated this was likely due to the fact that the basal level of LIFR expression in normal HTR8 cell line was too low.
Increased expression of MMP14 was involved in the release of sENG as well as in the LIFR-enhanced cell migration and invasion in vitro

To further confirm whether MMP14 is involved in the release of sENG and the invasiveness of HTR-8/SVneo cells, we inhibited MMP14 using either a specific inhibitor (NSC405020) or a specific siRNA. The efficacy of MMP14 knockdown by siRNA was demonstrated
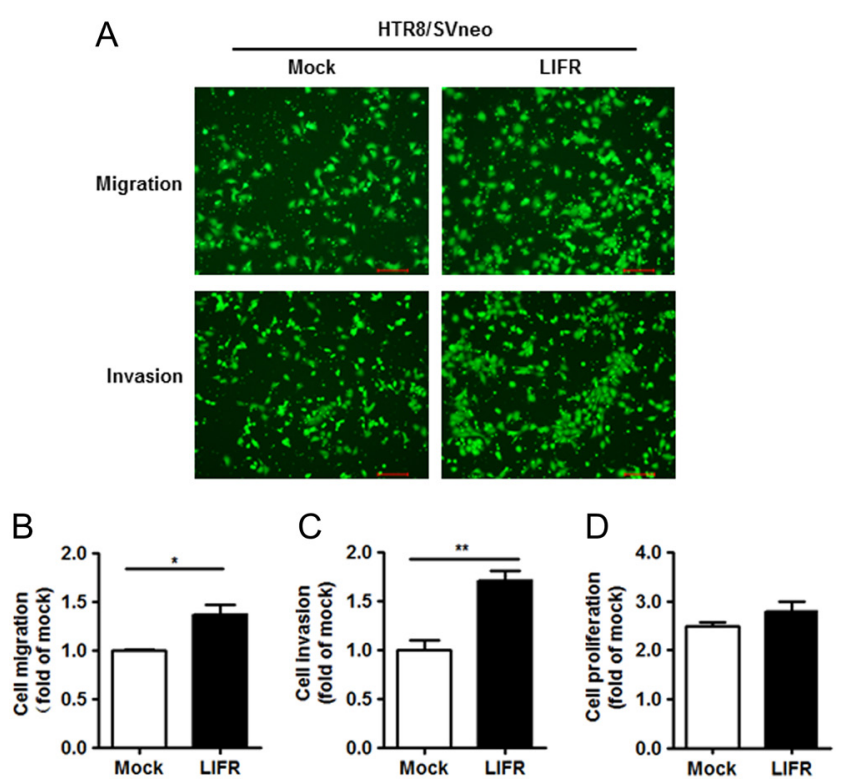

Figure 3 Overexpression of LIFR enhances the migration and invasion of HTR-8/SVneo cells. (A, B and C) Effects of the overexpression of LIFR on the migration and invasiveness of HTR-8/SVneo cells were determined using the Transwell system. (D) Effects of the overexpression of LIFR on HTR-8/SVneo cell proliferation by MTT assay. Scale bar $=200 \mu \mathrm{m}$. Bars represent the means \pm S.E.M. (Student's t-test, $* P<0.05, * * P<0.01)$. 

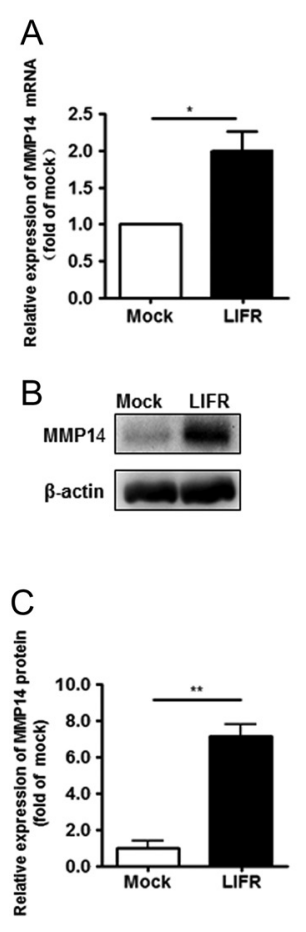

D
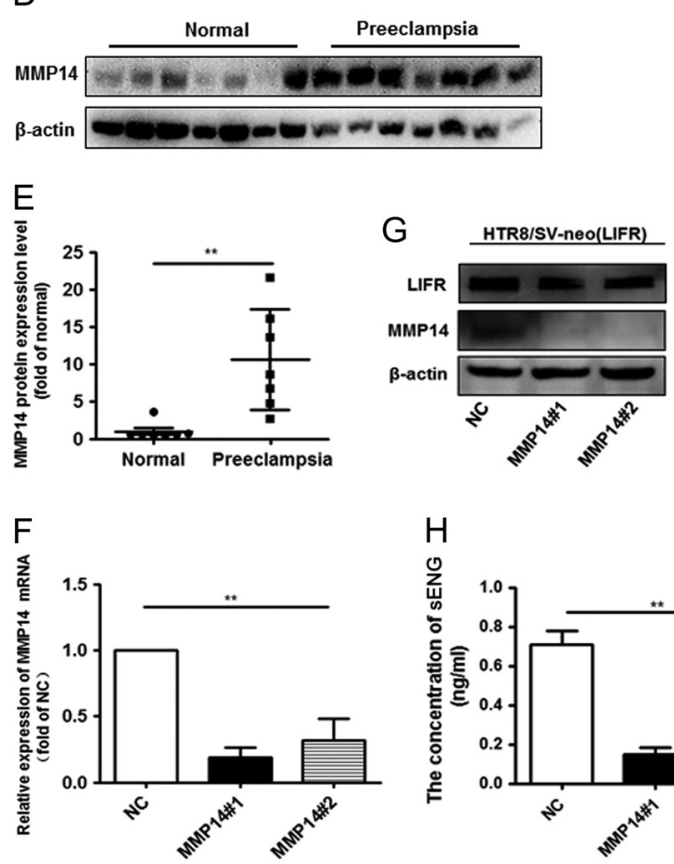

Figure 4 LIFR is involved in the release of SENG via the upregulation of MMP14 in HTR-8/Svneo cells. (A, B and C) The expressions of MMP14 mRNA and protein in HTR8/SVneo cells transfected with an expression vector encoding LIFR (LIFR) or an empty vector (Mock), according to qRT-PCR and Western blot. (D and E) The expression of MMP14 protein in preeclamptic and normal placentas, as determined by Western blot. ( $F$ and G) The efficacy of MMP14 siRNA was detected by Western blot and qRT-PCR in HTR8/SVneo cells after overexpression of LIFR. (H) The concentration of sENG in the cell supernatant after MMP14 knockdown was determined by ELISA in HTR8/SVneo cells overexpressing LIFR. The relative expression level of MMP14 protein was normalized to $\beta$-actin. The data are expressed as the means \pm S.E.M. Or S.D. (Student's t-test, one-way ANOVA, $\left.{ }^{*} P<0.05,{ }^{* *} P<0.01\right)$. by Western blot and qRT-PCR (Fig. 4F and G). The knockdown of MMP14 in HTR8/SVneo-LIFR cells significantly reduced sENG release compared with the negative control, as determined by ELISA (Fig. 4H). Meanwhile, NSC405020 robustly suppressed the LIFRinduced migration and invasion of HTR8/SVneo cells (Fig. 5).

These data confirmed that LIFR induced the release of sENG via an increase in MMP14 expression. In addition, an increase in MMP14 might contribute to the enhanced migration and invasion of HTR8/SVneo-LIFR cells.

\section{LIF, but not LIFR, increased cell migration and invasion through the activated STAT3 pathway}

It is well known that LIF is involved in various processes, including cell proliferation, differentiation and migration, through activation of the STAT3 pathway. In this study, we also found that different concentrations $(10-50 \mathrm{ng} / \mathrm{mL})$ of LIF significantly enhanced the migration and invasion of HTR8/SVneo and JAR cells (Fig. 6A, B, C and D), but their proliferation was not significantly affected (Fig. 6E); we also found that the enhanced cell migration
A

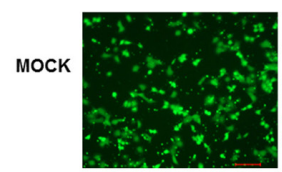

LIFR

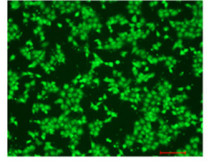

DMSO
Migration

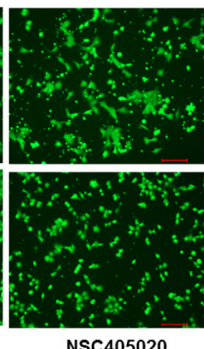

NSC405020

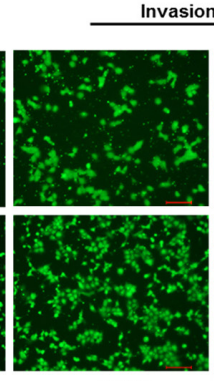

DMSO

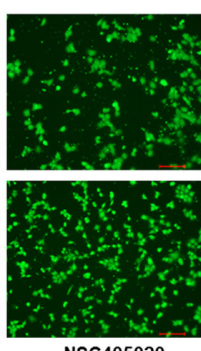

NSC405020
$\mathrm{B}$

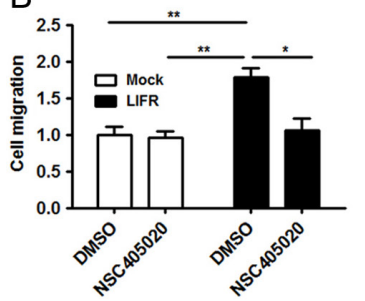

C

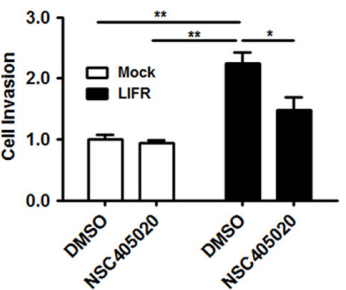

D

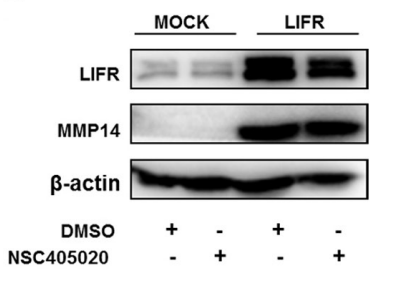

Figure 5 An MMP14 inhibitor (NSC405020) inhibits HTR-8/Svneo cell migration and invasion induced by LIFR. (A, B and C) The cell migration and invasion were decreased by NSC405020 as demonstrated by the Transwell system. (D) The expression of MMP14 protein in HTR8/SVneo cells treated with NSC405020 $(50 \mu \mathrm{m})$ was shown by Western blot. Scale bar $=200 \mu \mathrm{m}$. Bars represent the means \pm S.E.M. (Student's $t$-test, ${ }^{*} P<0.05,{ }^{* *} P<0.01$ ). 
A

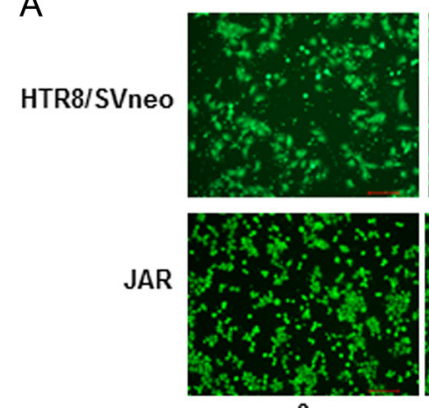

0

C

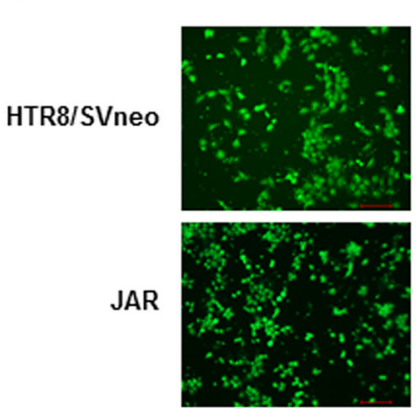

0
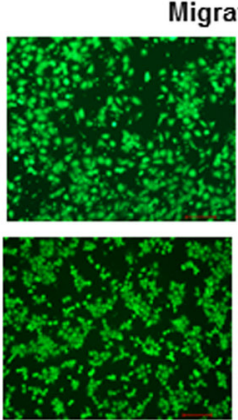

10
Migration

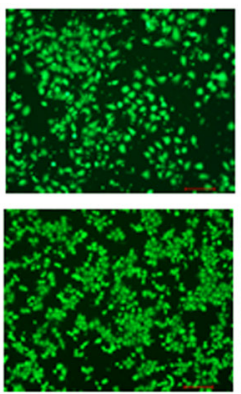

25

Concentration of LIF (ng/ml)

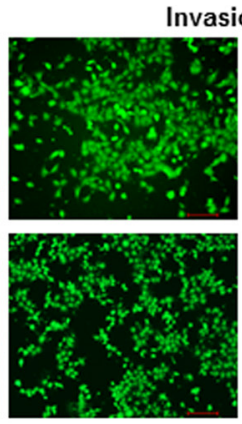

10

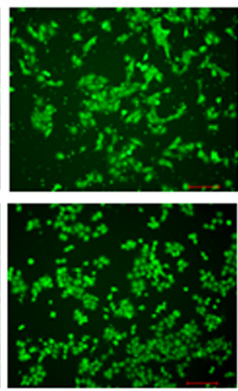

25
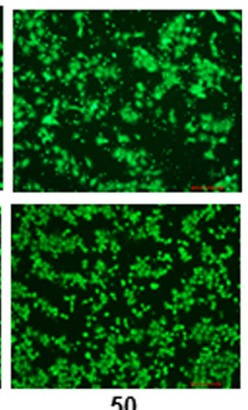

50

B

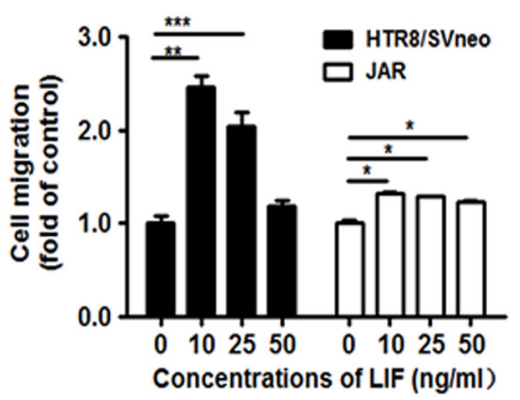

D

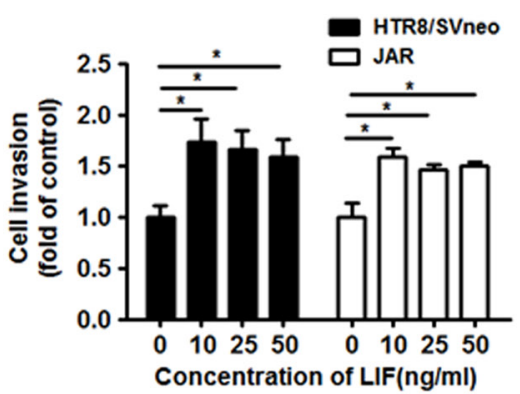

G

F

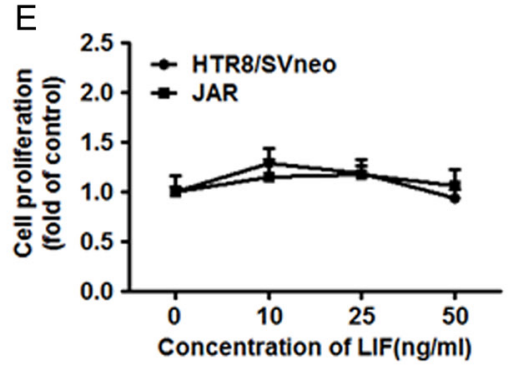

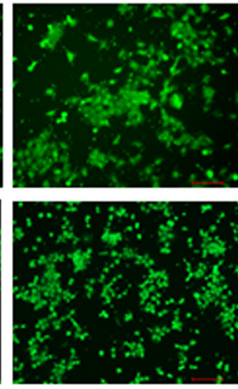

50
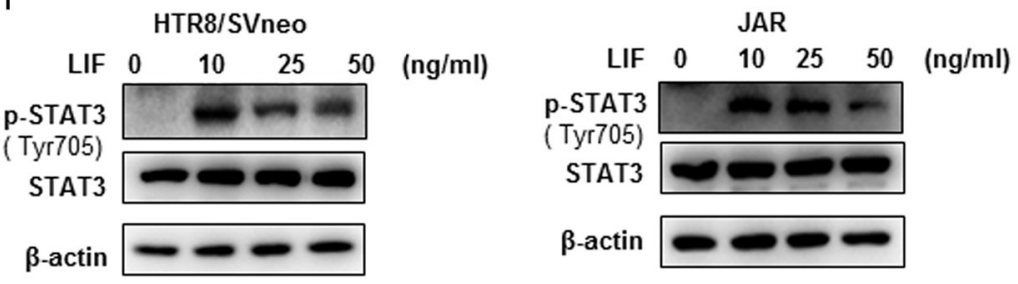

Figure 6 The exogenous LIF enhances the migration and invasion of JAR and HTR-8/SVneo cells through the phosphorylation of STAT3. (A, B, C, $\mathrm{D}$ and $\mathrm{E}$ ) The effects of LIF on cell migration, invasion and proliferation were measured by MTT assay and the Transwell system. (F and G) The phosphorylation of the STAT3 in JAR and HTR-8/SVneo cells after treated with different concentrations of LIF, as determined by Western blot. Scale bar $=200 \mu \mathrm{m}$. The bands represent STAT3, pSTAT3, and $\beta$-actin (internal reference) proteins, as shown by Western blot. The data are expressed as the means \pm S.E.M. (Student's $t$-test, $* P<0.05, * * P<0.01, * * * P<0.001$ ).

and invasion was mediated by activation of the STAT3 pathway (Fig. 6F and G) and the STAT3 inhibitor could decrease cell migration induced by LIF (Fig. 7). However, although LIFR overexpression enhanced cell migration and invasion, we did not observe that the overexpression of LIFR could stimulate the phosphorylation of STAT3 (Fig. 8).

\section{Discussion}

Preeclampsia (PE) is a gestational hypertensive syndrome and is a major cause of maternal mortality and morbidities, perinatal deaths, preterm births and intrauterine growth restriction (Sibai et al. 2005). Although much evidence has accumulated on the pathophysiology of preeclampsia, the accurate mechanism of this disorder remains to be determined. Previous studies have reported that LIFR expression was upregulated in vivo models of arterial injury and acute ocular hypertension (World et al. 2001, Hu et al. 2015), which provided evidence that LIFR might be associated with the occurrence of PE. In this study, we found that LIFR was upregulated in the placentas of women with PE and enhanced the release of sENG as well as the migration and invasion of HTR8/SVneo cells. We also demonstrated that MMP14 expression was increased by LIFR in HTR8/SVneo cells and was involved in the LIFR-enhanced release of sENG as well as cell migration and invasion.

In the current study, we first demonstrated that the expression of LIFR, but not that of LIF, was highly increased 


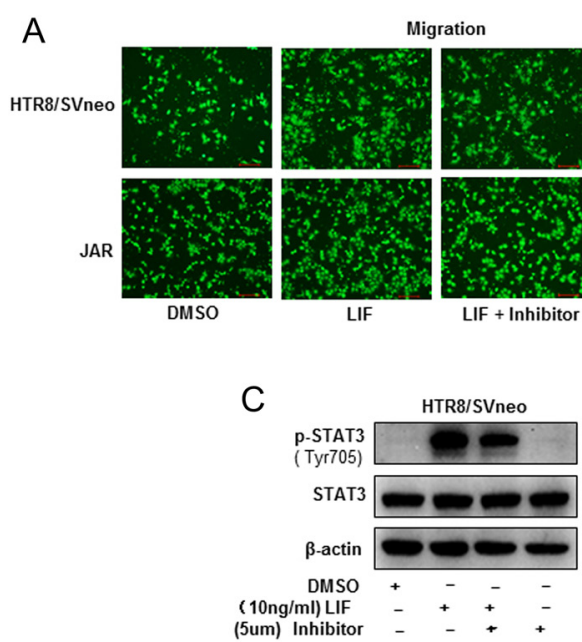

B

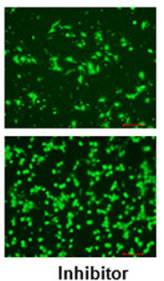

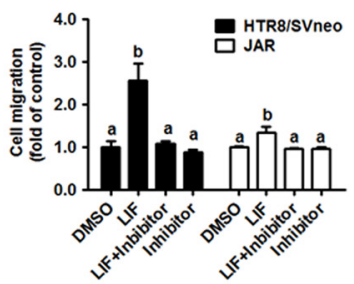

Figure 7 The STAT3 inhibitor can inhibit the migration of JAR and HTR-8/SVneo cells. (A and B) Effects of the STAT3 inhibitor on cell migration according to the Transwell system. (C and D) The expression of STAT3 and pSTAT3 in cells that were incubated with a STAT3 inhibitor according to Western blot. The data are normalized to $\beta$-actin. Scale bar $=200 \mu \mathrm{m}$. Bars represent the means \pm S.E.M. $a$ and $b$, means with different letters are significantly difference (one-way ANOVA, $* P<0.05)$. in the placentas of women with PE in comparison with the placentas of women with normal pregnancies. Carol and coworkers reported that LIFR was correlated with abnormal placental vasculature (Ware et al. 1995). These data suggested that such increase might be involved in the onset of preeclampsia. Importantly, our results also indicated that LIFR promoted sENG release as well as the migration and invasion of HTR8/SVneo cells. A previous study showed that high LIFR expression stimulated melanoma cell migration and was correlated with an unfavorable prognosis in melanoma (Guo et al. 2015). However, Luo and coworkers reported that LIFR acted as a suppressor of metastasis in hepatocellular carcinoma (Luo et al. 2015). The functions of LIFR in trophoblast cells need to be further investigated. The
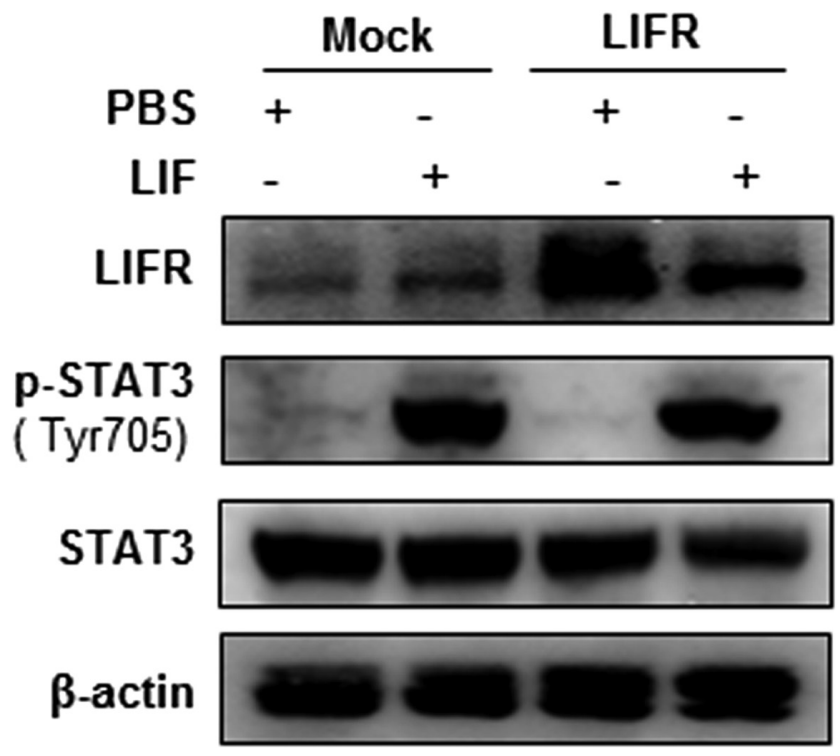

Figure 8 The overexpression of LIFR could not stimulate the phosphorylation of STAT3. The bands represent the LIFR, STAT3, pSTAT3 and $\beta$-actin (internal reference) proteins after Western blot analysis. soluble endoglin (sENG), which has been generally identified as a critical antiangiogenic factor, interacted with components of the TGF- $\beta / \mathrm{eNOS}$ and/or BMP-9 signaling pathways. This might result in endothelial dysfunction, which results from proteolytic shedding from membrane-bound endoglin in the circulation of women with preeclampsia (Li et al. 2001, Venkatesha 2006, Rathouska et al. 2015). In a murine model, the overexpression of human soluble endoglin resulted in a higher SBP in those mice compared with their wildtype littermates (Valbuena-Diez et al. 2012). Moreover, in our study, the increased sENG in the circulation of pregnant women with PE was consistent with a previous observation (Levine et al. 2006). More importantly, we found that after overexpression of LIFR in HTR8/SVneo cells, the release of sENG in the cell supernatant was significantly upregulated. These data provided evidence that LIFR was probably involved in the development of PE through the upregulation of sENG release, which directly resulted in endothelial cell dysfunction.

Another important finding of this study was that MMP14 expression was significantly increased by LIFR in HTR8/SVneo cells. It is well known that MMP14 plays an important role in cell migration and invasion. Recent studies indicated that MMP14 targeted membranebound endoglin to proteolytically release sENG (Noel et al. 2008, Hawinkels et al. 2010) and that MMP14 could directly degrade extracellular matrix components to regulate the invasiveness of various cells (Chen et al. 2016, Swayampakula et al. 2017). In the present study, the knockdown of MMP-14 strongly inhibited LIFRinduced SENG release in HTR-8 cells, whereas the inhibition of MMP14 suppressed the increased migration and invasion induced by LIFR in HTR8/SVneo cells. Moreover, the expression of MMP14 in the placentas of women with PE was significantly increased compared with placentas of women with normal pregnancies. Together, our observations indicated that increased expression of LIFR in PE may increase the release of sENG via the upregulation of MMP14. 
As it is known that the STAT3 pathway is closely related to the LIF/LIFR system, we also found that LIF stimulated the phosphorylation of the STAT3 pathway. However, LIFR could not activate the STAT3 pathway, which suggested that LIFR did not function through the STAT3 pathway in HTR8/SVneo cells. LIFR is an important receptor of several members of interleukin 6 family, such as leukemia inhibitory factor (LIF), oncostatin M (OSM), cardiotrophin 1 (CT1) and ciliary neurotrophic factor (CNTF) (Plun-Favreau et al. 2003, Hunt \& White 2016). It mediates many signaling pathways in different cells and tissues, including JAK/STAT3, MAPK/ERK1/2, MAPK/p38 and PI3K/AKT (Schiemann et al. 1995, Godoy-Tundidor et al. 2005, Magni et al. 2007). We cannot exclude the possibility that these pathways may be involved in the process of LIFR regulating MMP14 expression and sENG release. In addition, the expression level of ERK1/2 in HTR8/SVneo cells overexpressing LIFR was simply investigated. While we found that LIFR overexpression in HTR8 cells significantly activated the phosphorylation of ERK1/2(data not shown). We will continue to explore the mechanisms of LIFR-induced MMP14 expression and $\mathrm{SENG}$ release in the future.

In conclusion, our studies demonstrated that the LIFR protein level was significantly higher in the placentas of patients with PE compared with normotensive pregnancies. The overexpression of LIFR in HTR8/ SVneo cells strongly increased MMP14 expression and the release of $s E N G$, which is likely involved in the pathological mechanism of preeclampsia.

\section{Declaration of interest}

The authors declare that there is no conflict of interest that could be perceived as prejudicing the impartiality of the research reported.

\section{Funding}

This study was supported by Program for Outstanding Medical Academic Leader of Shanghai (to D T, No.77), the National Natural Science Foundation of China (No. 81471461 and No. 81771591), Program of Shanghai Subject Chief Scientist (No. 14XD1403200), and the Key Disciplines Group Construction Project of Pudong Health Bureau of Shanghai (No. PWZxq2014-02).

\section{References}

Chen W, Xia T, Wang D, Huang B, Zhao P, Wang J, Qu X \& Li X 2016 Human astrocytes secrete IL-6 to promote glioma migration and invasion through upregulation of cytomembrane MMP14. Oncotarget 7 62425-62438. (https://doi.org/10.18632/oncotarget.11515)

Chung J-Y, Song Y, Wang Y, Magness MR \& Zheng J 2004 Differential expression of vascular endothelial growth factor (VEGF), endocrine gland derived-VEGF, and VEGF receptors in human placentas from normal and preeclamptic pregnancies.. Journal of Clinical Endocrinology and Metabolism 89 2484-2490. (https://doi.org/10.1210/jc.2003-031580)
Dai CF, Jiang YZ, Li Y, Wang K, Liu PS, Patankar MS \& Zheng J 2011 Expression and roles of Slit/Robo in human ovarian cancer. Histochemistry and Cell Biology 135 475-485. (https://doi.org/10.1007/ s00418-011-0806-2)

Gallardo-Vara E, Blanco FJ, Roque M, Friedman SL, Suzuki T, Botella LM \& Bernabeu C 2016 Transcription factor KLF6 upregulates expression of metalloprotease MMP14 and subsequent release of soluble endoglin during vascular injury. Angiogenesis 19 155-171. (https://doi. org/10.1007/s10456-016-9495-8)

Godoy-Tundidor S, Cavarretta I, Fuchs D, Fiechtl M, Steiner H, Friedbichler K, Bartsch G, Hobisch A \& Culig Z 2005 Interleukin-6 and oncostatin $\mathrm{M}$ stimulation of proliferation of prostate cancer $22 \mathrm{Rv} 1$ cells through the signaling pathways of p38 mitogen-activated protein kinase and phosphatidylinositol 3-kinase. Prostate 64 209-216. (https://doi. org/10.1002/pros.20235)

Goldenberg RL, Culhane JF, lams JD \& Romero R 2008 Epidemiology and causes of preterm birth. Lancet 371 75-84. (https://doi.org/10.1016/ S0140-6736(08)60074-4)

Guo H, Cheng Y, Martinka M \& McElwee K 2015 High LIFr expression stimulates melanoma cell migration and is associated with unfavorable prognosis in melanoma. Oncotarget 6 25484-25498. (https://doi. org/10.18632/oncotarget.4688)

Hansen AR, Barne's CM, Folkman J \& McElrath TF 2010 Maternal preeclampsia predicts the development of bronchopulmonary dysplasia. Journal of Pediatrics 156 532-536. (https://doi.org/10.1016/j. jpeds.2009.10.018)

Hawinkels LJ, Kuiper P, Wiercinska E, Verspaget HW, Liu Z, Pardali E, Sier CF \& ten Dijke P 2010 Matrix metalloproteinase-14 (MT1-MMP)mediated endoglin shedding inhibits tumor angiogenesis. Cancer Research 70 4141-4150. (https://doi.org/10.1158/0008-5472.CAN-094466)

Ho L, van Dijk M, Chye STJ, Messerschmidt DM, Chng SC, Ong S, Yi LK, Boussata S, Goh GH, Afink GB et al. 2017 ELABELA deficiency promotes preeclampsia and cardiovascular malformations in mice. Science $\mathbf{3 5 7}$ 707-713. (https://doi.org/10.1126/science.aam6607)

Hu Q, Huang C, Wang Y \& Wu R 2015 Expression of leukemia inhibitory factor in the rat retina following acute ocular hypertension. Molecular Medicine Reports 12 6577-6583. (https://doi.org/10.3892/ mmr.2015.4287)

Hunt L \& White J 2016 The role of leukemia inhibitory factor receptor signaling in skeletal muscle growth, injury and disease. Advances in Experimental Medicine and Biology 900 45-59. (https://doi. org/10.1007/978-3-319-27511-6_3)

Jiang Y Wang K, Fang R \& Zheng J 2010 Expression of aryl hydrocarbon receptor in human placentas. Journal of Histochemistry and Cytochemistry 58 679-685. (https://doi.org/10.1369/jhc.2010.955955)

Jeyabalan A, McGonigal S, Gilmour C, Hubel CA \& Rajakumar A 2008 Circulating and placental endoglin concentrations in pregnancies complicated by intrauterine growth restriction and preeclampsia. Placenta 29 555-563. (https://doi.org/10.1016/j.placenta.2008.03.006)

Levine RJ, Lam C, Qian C, Yu KF, Maynard SE, Sachs BP, Sibai BM, Epstein FH, Romero R, Thadhani R et al. 2006 Soluble endoglin and other circulating antiangiogenic factors in preeclampsia. New England Journal of Medicine 355 992-1005. (https://doi.org/10.1056/NEJMoa055352)

Li C, Guo B, Bernabeu C \& Kumar S 2001 Angiogenesis in breast cancer: the role of transforming growth factor beta and CD105. Microscopy Research and Technique 52 437-449. (https://doi.org/10.1002/10970029(20010215)52:4<437::AID-JEMT1029>3.0.CO;2-G)

Luo Q, Wang C, Jin G, Gu D, Wang N, Song J, Jin H, Hu F, Zhang Y, Ge T et al. 2015 LIFR functions as a metastasis suppressor in hepatocellular carcinoma by negatively regulating phosphoinositide 3-kinase/AKT pathway. Carcinogenesis 36 1201-1212. (https://doi.org/10.1093/carcin/ bgv108)

Luo Q, Zhang Y, Wang N, Jin G, Jin H, Gu D, Tao X, Huo X, Ge T, Cong W et al. 2014 Leukemia inhibitory factor receptor is a novel immunomarker in distinction of well-differentiated HCC from dysplastic nodules. Oncotarget 6 6989-6899.

Magni P, Dozio E, Ruscica M, Watanobe H, Cariboni A, Zaninetti R, Motta M \& Maggi R 2007 Leukemia inhibitory factor induces the chemomigration of immortalized gonadotropin-releasing hormone neurons through the independent activation of the Janus kinase/signal transducer and activator of transcription 3, mitogen-activated protein 
kinase/extracellularly regulated kinase $1 / 2$, and phosphatidylinositol 3-kinase/Akt signaling pathways. Molecular Endocrinology 21 1163-1174. (https://doi.org/10.1210/me.2006-0270)

McGinnis R, Steinthorsdottir V, Williams NO, Thorleifsson G, Shooter S, Hjartardottir S, Bumpstead S, Stefansdottir L, Hildyard L, Sigurdsson JK et al. 2017 Variants in the fetal genome near FLT1 are associated with risk of preeclampsia. Nature Genetics 49 1255-1260. (https://doi. org/10.1038/ng.3895)

Mol BWJ, Roberts CT, Thangaratinam S, Magee LA, de Groot CJM \& Hofmeyr GJ 2016 Pre-eclampsia. Lancet 387 999-1011. (https://doi. org/10.1016/S0140-6736(15)00070-7)

Noel A, Jost M \& Maquoi E 2008 Matrix metalloproteinases at cancer tumor-host interface. Seminars in Cell and Developmental Biology 19 52-60. (https://doi.org/10.1016/j.semcdb.2007.05.011)

Plun-Favreau H, Perret D, Diveu C, Froger J, Chevalier S, Lelièvre E, Gascan H \& Chabbert M 2003 Leukemia inhibitory factor (LIF), cardiotrophin-1, and oncostatin $M$ share structural binding determinants in the immunoglobulin-like domain of LIF receptor. Journal of Biological Chemistry 278 27169-27179. (https://doi.org/10.1074/jbc. M303168200)

Rathouska J, Jezkova K, Nemeckova I \& Nachtigal P 2015 Soluble endoglin, hypercholesterolemia and endothelial dysfunction. Atherosclerosis $\mathbf{2 4 3}$ 383-388. (https://doi.org/10.1016/j.atherosclerosis.2015.10.003)

Redman CW \& Sargent IL 2005 Latest advances in understanding preeclampsia. Science 308 1592-1594. (https://doi.org/10.1126/ science.1111726)

Schiemann WP, Graves LM, Baumann H, Morella KK, Gearing DP, Nielsen MD, Krebs EG \& Nathanson NM 1995 Phosphorylation of the human leukemia inhibitory factor (LIF) receptor by mitogenactivated protein kinase and the regulation of LIF receptor function by heterologous receptor activation. PNAS 92 5361-5365. (https://doi. org/10.1073/pnas.92.12.5361)

Sibai B, Dekker G \& Kupferminc M 2005 Pre-eclampsia. Lancet 365 785-799. (https://doi.org/10.1016/S0140-6736(05)71003-5)

Souza JP, Gülmezoglu AM, Vogel J, Carroli G, Lumbiganon P, Qureshi Z, Costa MJ, Fawole B, Mugerwa Y, Nafiou I et al. 2013 Moving beyond essential interventions for reduction of maternal mortality (the WHO Multicountry Survey on Maternal and Newborn Health): a crosssectional study. Lancet 381 1747-1755. (https://doi.org/10.1016/S01406736(13)60686-8)

Swayampakula M, McDonald PC, Vallejo M, Coyaud E, Chafe SC, Westerback A, Venkateswaran G, Shankar J, Gao G, Laurent EMN et al. 2017 The interactome of metabolic enzyme carbonic anhydrase IX reveals novel roles in tumor cell migration and invadopodia/MMP14mediated invasion. Oncogene 36 6244-6261. (https://doi.org/10.1038/ onc.2017.219)

Valbuena-Diez AC, Blanco FJ, Oujo B, Langa C, Gonzalez-Nunez M, Llano E, Pendas AM, Diaz M, Castrillo A \& Lopez-Novoa JM 2012 Oxysterol-induced soluble endoglin release and its involvement in hypertension. Circulation 126 2612-2624. (https://doi.org/10.1161/ CIRCULATIONAHA.112.101261)

Venkatesha S, Toporsian M, Lam C, Hanai J, Mammoto T, Kim YM, Bdolah Y, Lim KH, Yuan HT, Libermann TA et al. 2006 Soluble endoglin contributes to the pathogenesis of preeclampsia. Nature Medicine 12 642-649. (https://doi.org/10.1038/nm1429)

Wang K, Song Y, Chen DB \& Zheng J 2008 Protein phosphatase 3 differentially modulates vascular endothelial growth factor- and fibroblast growth factor 2-stimulated cell proliferation and signaling in ovine fetoplacental artery endothelial cells. Biology of Reproduction 79 704-710. (https://doi.org/10.1095/biolreprod.108.068957)

Ware CB, Horowitz MC, Renshaw BR, Hunt JS, Liggitt D, Koblar SA, Gliniak BC, McKenna HJ, Papayannopoulou T, Thoma B et al. 1995 Targeted disruption of the low-affinity leukemia inhibitory factor receptor. Development 121 1283-1299.

World CJ, Rolfe BE \& Campbell JH 2001 Regulation of LIF receptor expression in vascular smooth muscle. Annals New York Academy of Sciences 947 323-328. (https://doi.org/10.1111/j.1749-6632.2001. tb03956.x)

Zeisler H, Llurba E, Chantraine F, Vatish M, Staff AC, Sennstrom M, Olovsson M, Brennecke SP, Stepan H, Allegranza D et al. 2016 Predictive value of the sFlt-1:PIGF ratio in women with suspected preeclampsia. New England Journal of Medicine 374 13-22. (https://doi.org/10.1056/ NEJMoa1414838)

Zheng Q, Dai K, Cui X, Yu M, Yang X, Yan B, Liu S \& Yan Q 2016 Leukemia inhibitory factor promote trophoblast invasion via urokinase-type plasminogen activator receptor in preeclampsia. Biomed Pharmacother 80 102-108. (https://doi.org/10.1016/j.biopha.2016.03.005)

Received 21 September 2017

First decision 20 October 2017

Revised manuscript received 30 November 2017

Accepted 22 January 2018 